\title{
La capilla musical de la Catedral de Toledo durante el primer decenio del siglo XVII
}

\author{
Musical Chapel in Cathedral of Toledo during the \\ first decade of the $17^{\text {th }}$ Century
}

\author{
Juan Bautista Canadillas Gómez \\ Universidad de Castilla-La Mancha \\ juanbautista.canadillas@alu.uclm.es
}

\section{RESUMEN}

El presente texto estudia la estructura, el funcionamiento y los problemas de la capilla de música de la catedral de Toledo para ofrecer una visión global de su situación al inicio del siglo XVII. Observando a los maestros de capilla se mostrará como aún en los periodos en los cuales no existía uno la catedral estaba bien nutrida de individuos competentes que podrían suplir sus funciones. Al hablar de los organistas se reflexionará a cerca de las posibles diferencias al emplear los términos "músico de tecla" y "organista". Al respecto de los instrumentistas se mencionará a aquellos que sirvieron a la catedral de forma esporádica. Además se indagará en los salarios de la capilla, sus permisos y también en su supuesta conflictividad.

Palabras clave: Maestro de capilla, Cantor, Organista, Ministril, Seise, Músico de tecla, Racionero, Cabildo, Toledo.

\begin{abstract}
This paper analyses the structure, functioning and problems of the music chapel of the Toledo Cathedral in order to provide an overview of its situation at the beginning of the seventeenth century. The study of the chapel masters will show that, even in periods when the cathedral was uninhabited, their duties were filled by competent individuals. The terminology, e.g. possible differences between a "key musician" and an "organist", will be put under examination while analysing the organists. In addition, the instrumentalists who served the cathedral sporadically, will be briefly reviewed. Finally, the wages of the chapel, its permits and also its alleged conflict will be discussed.
\end{abstract}




\section{LA CAPILla MUSical de la CATEDRAL DE TOLEDO DURANTE EL PRIMER DECENIO DEL SIGLO XVII}

Key words: Chapel Master, Chanter, Organist, Minstrel, Choir Boy, Prebedary, Chapter, Toledo.

Canadillas Gómez, J. B. (2016). La capilla musical de la Catedral de Toledo durante el primer decenio del siglo XVII. Cuadernos de Investigación Musical, 1, 128-149.

doi: dx.doi.org/10.18239/invesmusic_2016.01.1312

\section{INTRODUCCIÓN}

El presente artículo muestra, de manera sintetizada, las cuestiones investigadas en el trabajo final para la obtención del Máster en Investigación e Innovación Musical cursado en la Universidad de Catsilla-La Mancha y presentado en el año 2015 titulado de la misma manera que este trabajo. La intención perseguida fue indagar en la situación de una de las grandes capillas musicales españolas en un tiempo "a caballo" entre el renacimiento y el barroco ayudando a llenar las lagunas existentes en el estudio del siglo XVII; también ofrecer información novedosa al respecto de músicos del periodo, algunos conocidos y otros no tanto, que sirvieron en esta catedral, y por último recuperar alguna de las obras existentes que pudiese adscribirse a la cronología estudiada.

En cuanto al estado de la cuestión, además de las obras más antiguas como las de Felipe Rubio Piqueras, que recuperan toda la información posible sobre sus respectivos temas, hay dos trabajos que encuadran este periodo. Por un lado la obra de François Reynaud, La polyphonie tolédane et son milieu, des premiers témoignages aux environs de 1600, que pondría el limite inferior, y la de Carlos Martínez-Gil, La capilla de música de la catedral de Toledo (1700-1764): Evolución de un concepto sonoro, que marcaría el superior. Existe además un Trabajo Fin de Máster acerca del maestro de capilla Tomás Micieces (1650-1662) presentado en el Máster en Patrimonio Histórico: Investigación y Gestión de la Universidad de Castilla-La Mancha en el año 2013. A estas obras se debe añadir una más, la tesis doctoral presentada en el año 2016 en la Universidad Autónoma de Madrid por Natalia Medina Hernández titulada La vida musical en la catedral de Toledo durante el siglo XVII y que supone un gran paso a la hora de rellenar el conocimiento sobre este periodo.

\section{La Capilla Musical de la Catedral Primada de España}

Existen al menos cuatro fuentes que nos hablan de cómo se encontraría conformada la capilla musical de esta catedral; la primera de estas son Relaciones topográficas de los pueblos de España, hechas de orden de Felipe II donde en el capítulo 49 se examina la composición de los coros de la catedral. Referente a la música es importante decir que refleja la presencia de 12 cantores, 8 cantores racioneros, 8 ministriles, 4 organistas, 2 chantres, 2 sochantres, 2 entonadores de órganos además de 6 seises (Viñas Mey \& Paz, 


\section{JUAN BAUTISTA CANADILLAS GÓMEZ}

1951: 534-535). Por su parte Alcoçer en su obra Hystoria, o descripción de la Imperial Cybdad de Toledo del año 1554 realiza una interesante mención a la existencia de 52 racioneros en la catedral para la fecha de creación de su escrito, de los cuales, 12 tendrían funciones directamente relacionadas con la actividad musical, eran: 1 organista, 1 maestro de capilla, 2 sochantres/socapiscoles y 8 cantores; también habla de la existencia de 10 cantores extraordinarios (Reynaud, 1996). La tercera de las fuentes es sin embargo la más antigua de todas ellas, procede de una obra de 1549 llamada Summi Templi Toletani Descriptio de Blas de Ortiz canónigo de la catedral. Él en su descripción refleja la existencia de: un maestro de música, 8 cantores con ración, otros 12 cantores que percibían salario en base a su habilidad y voz, 2 sochantres, 1 ayudante de sochantre, un encargado de poner los libros en el coro, 8 ministriles, un organista, un organista sustituto y un insuflador de órganos (Martínez Gil, 2003: 50). La última de las fuentes es una obra del año 1757, llevada a cabo por Juan Bravo de Acuña y titulada Descripción de la Santa Primada de Toledo donde el autor divide la composición de la capilla musical en los siguientes puestos: un maestro de capilla, 2 sochantres, un claustrero, 3 organistas, 3 entonadores de órganos, 11 ministriles, otros 2 músicos de tecla y otros 11 salmeadores asalariados (Martínez Gil, 2003: 50).

Como se puede observar en cuatro fuentes diferentes, tres de ellas próximas temporalmente encontramos una disparidad considerable, si bien existen elementos en común, como la existencia de 8 cantores racioneros además de otros 12 "extraordinarios", dos sochantres o la existencia temprana de una "capilla de ministriles". Esta capilla de ministriles nunca estuvo asociada a un maestro de ministriles como sí ocurrió en otras localizaciones del país como León (Álvarez Pérez, 1959: 53).

Para el año 1600, punto de inicio de este trabajo la estructura de la capilla musical sería la siguiente: 
La CAPILla MUSiCAL DE la CATEDRAL DE TOLEdo DURANTE EL

PRIMER DECENIO DEL SIGLO XVII

$\begin{array}{lll}\text { Cargo } & \text { Posición } & \text { Número } \\ \text { Chantre/Capiscol } & \text { Dignidad } & 1 \\ \text { Músico de tecla } & \text { Racionero } & 1 \\ \text { Maestro de capilla } & \text { Racionero } & 1 \\ \text { Cantores } & \text { Racioneros } & 7 \text { (una vacante) } \\ \text { Sochantres/Socapiscoles } & \text { Racioneros } & 2 \\ \text { Maestro de música del } & \text { Racionero } & 1 \\ \text { Colegio de los } & & \\ \text { Infantes/claustrero } & & \\ \text { Músico de tecla } & \text { Asalariado } & 1 \\ \text { Cantores } & \text { Asalariados } & 13 \\ \text { Salmeadores } & \text { Asalariados } & 7 \\ \text { Ayudante de sochantre } & \text { Asalariado } & 1 \\ \text { Ministriles } & \text { Asalariados } & 10 \\ \text { Seises } & - & \text { Número variable } \\ \text { Organista } & \text { Asalariado } & 1 \\ \text { Entonador de órganos } & \text { Asalariado } & 1 \\ \text { Guarda de libros } & \text { Asalariado } & 1 \\ \text { Encargado de poner los } & \text { Asalariado } & 1 \\ \text { libros en el coro } & & 2 \\ \text { Libreros } & \text { Asalariados } & \end{array}$

Tabla 1.

Es una evidencia que los puestos de trabajo directa o indirectamente relacionados con la actividad musical en el templo son numerosos y variables; este hecho se puede deber entre otras muchas cosas a que algún puesto se encontrase vacante por la muerte o marcha de algún racionero o asalariado. Es necesario aludir también a que la ambivalencia de algunos de los trabajos, como ayudante de sochantre puede inducir a considerar que la capilla era ligeramente más amplia de lo que realmente fue.

\section{El MAestro de Capilla}

La primera vez que aparece el término en las fuentes de la sede toledana es en el Libro tercero de Prebendas, adjunto a la ración $\mathrm{n}^{\circ} 35$, bajo el título de maestro de capilla. Aparecen además una serie de nombres que se remontan hasta 1460. Un inconveniente a esta lista es que algunos racioneros se encargaron durante largos periodos de tiempo de ser maestros de capilla sin poseer la dicha ración. 


\section{JUAN BAUTISTA CANADILLAS GÓMEZ}

En cuanto a las actividades de las que se encargaría este racionero de la sede toledana encontramos varias fuentes que las detallan; una es el Libro de Relaciones, en su página 25, donde aparecen descritas las ocupaciones que debía de llevar a cabo (Rubio Piqueras, 1922: 203) y otra las Actas Capitulares del 20 de septiembre de 1593, donde se citan de forma detallada las funciones que tiene que cumplir el poseedor del magisterio de capilla relativas a los seises (Martínez Gil, 2003: 117).

\subsection{Alonso LoBo}

La primera cuestión compleja a la que nos enfrentamos al hablar de este maestro de capilla es su propio nombre, como menciona Inmaculada Cárdenas (1987: 12) se han encontrado dos nombres haciendo referencia a él, Alonso y Alfonso, lo cual llevó a algunos investigadores en la primera mitad del siglo XX ha considerar que podían ser dos individuos diferentes; además, comparada esta cuestión con la de Alonso de Tejeda, su tocayo y sucesor en el cargo de la sede toledana, se puede afirmar que la ambivalencia de la denominación era más o menos usual.

El 22 de marzo de 1593 utilizando sus días, el maestro Boluda partió de Toledo para entrar al servicio del cardenal; tras esto se promulgará el tradicional edicto que dará 60 días para presentarse en oposición; sin embargo ante la falta de examinantes se toma la decisión de prorrogarlo, al mes de julio. Pocos días después se presenta el maestro de capilla de León, Alonso de Tejeda. El 7 de septiembre se convoca a la capilla para examinar a otro candidato, Alonso Lobo. El examen tuvo lugar el 16 y no se precisa si los demás músicos de la catedral estuvieron. El día 20 de septiembre el cabildo elige por voto secreto a Alonso Lobo, al cual demanda que se presente para su ración. El día 6 de Octubre se encarga al licenciado Baltasar de Avilés la realización del pertinente informe de limpieza de sangre. Seis días después el maestro solicita un aumento de su salario que el cabildo acepta, pasando a ganar 200 ducados. El 26 de noviembre es admitido como racionero y el 7 de Diciembre toma posesión (Reynaud, 1996: 133).

Las obligaciones presentadas por Alonso Lobo, así como sus licencias son exactamente las mismas que poseyó su antecesor inmediato. Aparecen con cierta frecuencia en las actas permisos para ausentarse de Toledo, como por ejemplo el de doce días que tomó para ir al monasterio de Guadalupe junto con otros cantores (Reynaud, 1996: 133). Respecto a los permisos, en general es interesante reseñar que de entre las múltiples razones por las que le eran concedidos a los maestros de capilla no aparece ninguna mención a que les ofreciesen con el motivo de componer obras para la catedral, al contrario de lo que ocurre en otras catedrales españolas (Capdepón Verdú, 2013: 194). En el año 1600 encontramos otro viaje, en este caso por necesidades de la capilla en busca de cantores y por el que originalmente se le dará un pago de 40.000 maravedís. Sabemos también por noticias de este año 1600 que por la creación de los villancicos navideños originalmente se le daban 1500 maravedís, pero que de aquí en adelante esa cantidad se dobló (Reynaud, 1996: 133) y permaneció constante también para sus sucesores.

Cuando Alonso Lobo ocupa el magisterio de capilla de la catedral de Toledo lo hizo en posesión de una ración de cantor (tenor). Por ello el cabildo solicita en 1599 a Roma que expida una bula que le permita anexionar estos cargos a una de las raciones vacantes en el 


\section{La CAPILla MUSiCAL DE la CATEDRAL DE TOLEdo DURANTE EL PRIMER DECENIO DEL SIGLO XVII}

momento. La bula se presenta en septiembre de 1600 y además de la del magisterio de capilla se presenta otra para un cantor (Reynaud, 1996: 134). El 15 de diciembre el cardenal le concede la ración de maestro de capilla y le permite guardar la antigüedad que poseía en la otra ración de cantor, la cual quedará vacante. El 21 de enero de 1604 tomó sus días de recreación y no volvió a ocupar su puesto, tras esta marcha encontramos algunas alusiones al maestro, una de ellas relacionada con cuando comenzó a cobrar en la catedral de Sevilla, y la otra es un libramiento de 4532 maravedís por los alimentos de los seises de Enero de 1604 (Acta Capitular del Cabildo de Toledo, Vol. 23, AC de. 24-7-1605 Fol. 207v).

\subsubsection{Obras de Alonso Lobo durante su estancia en Toledo}

- Liber primus Missarum, compuesto por 6 misas y 5 motetes y 2 antífonas a la Virgen que fue investigado y transcrito (al menos parcialmente) por Robert Stevenson (1961: 268)

- Códice Polifónico $n^{\circ} 22$ se conservan 2 pasiones de este autor, además de un fabordón y otra pasión que abre el libro y que aparece sin autoría (Rubio Piqueras, 1924, pp. 71-72) pero que Reynaud considera que podría pertenecer al de Osuna (Reynaud, 1996: 135).

- $\quad$ Responso Libera me domine para el día de los finados a 5 voces, conservado en el Códice Polifónico n²4 (Rubio Piqueras, 1924: 75).

- Una serie de obras recogidas en el Códice Polifónico n 25, donde encontramos 10 lamentaciones, 9 salmos, 2 himnos de dedicación eclesiástica, 2 himnos para todos los santos, 3 himnos a San Eugenio, 7 te deum laudamus, 2 himnos de navidad, y 2 himnos sin ninguna advocación concreta (Rubio Piqueras, 1924: 75)*.

- Anima mea dominum encontrado en el año 2010 en el fondo Reserva del Instituto Español de Musicología por Bernadette Nelson (2010).

- Un posible Magnificat para dos coros encontrado "a papeles sueltos" en el Archivo Musical Moderno de la catedral, en la parte superior donde normalmente se señala la autoría únicamente pone Alfonso; lo cual nos lleva a la problemática de quien pudo ser su compositor, siendo las dos opciones más plausibles Alonso Lobo y Alonso de Tejeda, ambos maestros en Toledo a comienzos del siglo XVII.

\subsection{JORGE DE SANTA MARÍA}

Este músico desempeñó su labor durante toda su vida en la primada toledana; desde su estancia como seise bajo el magisterio de Bernardino de Rivera hasta su fallecimiento Durante su largo periplo por la catedral se encargó en numerosas ocasiones de la búsqueda, cuidado y examinación de los seises; la selección de repertorio; también

\footnotetext{
${ }^{*}$ Por las condiciones en las que se encontraba Michael Noone solo pudo recuperar una de las obras del maestro Lobo.

${ }^{\dagger}$ Obra transcrita en el Trabajo Fin de Master que precede a este artículo.
} 


\section{JuAN BAUTISTA CANADILLAS GÓMEZ}

realizó donaciones de libros de canto de su propiedad (Reynaud, 1996: 125-126), inventarios de los libros de música que poseía la capilla y fue en la práctica un maestro de capilla extraoficial en los periodos en los que los maestros presentes no podían ejercer sus funciones o en los que no había uno para llevarlas a cabo.

Adquirió su ración en el año 1581, y en este puesto lo encontramos activo al comienzo del siglo como señalan claramente los libros de Obra y Fábrica donde se hace relación de que continúa recibiendo el cobro de su ración. El año 1604 tras la decisión de marcharse de Alonso Lobo, el cabildo le encarga el cuidado de los seises (ACCT, Vol. 23, AC de 28-01-1604, Fol. 146r). Durante este tiempo la actividad relativa a la dirección musical no será llevada a cabo por el citado racionero, sino por otro cantor veterano, Pedro de los Ríos. Podríamos asumir entonces que mientras uno desarrollaría las actividades de dirección artística el otro se encargaría de las labores de enseñanza y composición. Durante el comienzo de las oposiciones para encontrar al nuevo maestro que se encargue de la capilla, se gratifica a Jorge de Santa María para que pueda proveer comida a los seises, además se le insta a que reciba a un seise que originalmente se había llevado Alonso Lobo con él a Sevilla (ACCT, Vol. 23, AC de 29-05-1604, Fol. 184r.).

Alonso de Tejeda, por diversas razones no tomó posesión hasta Mayo de 1605 por lo cual Santa María durante todo este tiempo continuó cuidando y enseñando a los niños cantores, como demuestran las libranzas en la Obra y Fábrica dirigidas a él por el cuidado de los seises (ACCT. Vol. 23, AC de 26-01-1605, Fol. 257r.). En el año 1606 encontramos dos informaciones en apariencia contradictorias, por un lado, en los libros de Obra y Fábrica se dice que desde el año 1606 se encargó del cuidado de los seises el maestro Alonso de Tejeda, pero una noticia del 11 de noviembre del mismo año da a entender que hasta esa fecha estuvieron a cargo del racionero Santa María (ACCT, Vol. 24, AC de 11-11-1606, Fol. 91r.).

El año 1607 se abre con una propuesta recogida en las Actas Capitulares y que atañe a la ración que ocupa este racionero; se propone en el cabildo que a la dicha ración se adhiera el magisterio de ceremonias (ACCT, Vol. 24, AC de 08-01-1607, Fol. 101v); el 17 de marzo aparece una nueva noticia al respecto y desde ese momento no aparecerán más menciones; los libros de Obra y Fábrica tampoco reflejan ninguna modificación en su ración por lo que se puede concluir que no llegó a ocurrir.

La última noticia del racionero toledano es del día 20 de diciembre de 1608 cuando el cabildo le concede una licencia de mañanas desde el dicho día hasta el "fin de tercio" ACCT, Vol. 24, AC de 20-12-1608, Fol. 253v). Aún con la ausencia de noticias suyas durante el año 1609 los libros de Obra y Fábrica informan de que continuó cobrando su ración con total normalidad.

\footnotetext{
${ }^{\ddagger}$ Serían 3 el de fin de Abril, el de fin de Agosto y el de fin de Diciembre.
} 


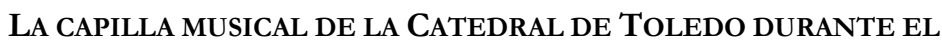 PRIMER DECENIO DEL SIGLO XVII}

\subsubsection{Las composiciones de Jorge de Santa María}

- Fondo Reserva Ms1: calenDa de nro pe s.tiago a 4; calenDa Del uautista a 4 Fol.40v41; calenDa De san juan eban/gelista; calenda de navidat (Nelson, 2010)

- Códice Polifónico 22: Feria III Maioris Hoeb; Feria IV Maioris Hoebdomadoes.

\subsection{Alonso de TejedA}

En el año 1604, transcurrido el tiempo establecido desde la emisión del edicto de ración, y durante el cual se consideraron cuales serían las pruebas más idóneas para demostrar la valía de los aspirantes (Preciado, 1974: 76), se procede a examinar a los aspirantes, las evaluaciones comenzaron el día 21 de mayo y el día 31 se decidió suspender las pruebas por dos meses ${ }^{* *}$.

El 28 y 29 de julio prosiguen las dichas pruebas de aptitud, siendo examinado Alonso de Tejeda. El 31 de Julio se convoca a los cantores para que todos y cada uno de ellos diese opinión sobre los 5 candidatos; el vencedor fue Alonso de Tejeda. El 12 de Noviembre se analizó y dio por bueno el informe de limpieza de sangre y se le otorgó la ración y plaza de maestro de capilla; sin embargo al no encontrarse en Toledo ni haber dejado a nadie con un poder para recibir posesión en su nombre fue llamado para tomar su nuevo puesto, cosa que no ocurrió hasta 6 meses después el 15 de abril de 1605.

Quizá la cuestión más llamativa que lo atañe directamente durante los límites temporales del trabajo es que en 1606, volvió a ejercer como maestro de capilla de Zamora y envió una carta al cabildo solicitándole un puesto para su cuñado; sin embargo prontamente fue llamado al orden, instándole a que se decidiese a tomar su cargo o que renunciase; decidió regresar (Preciado, 1974: 67).

En términos generales el cabildo toledano estuvo muy a gusto con el citado maestro de capilla ya que era una persona calmada y que ejercía sus labores con la dignidad y eficiencia exigida. Muy poco después de comenzar a ejercer recibió un aumento de salario que hizo que pasase a cobrar 75.000 maravedís; recibió además remuneraciones por escribir los villancicos y algún extra debido a su buena disposición y a su habilidad para el canto (Preciado, 1974: 81). Para el año en el que finaliza esta investigación, continúa ejerciendo las labores habituales relacionadas con su puesto.

\subsubsection{Obras de su estancia en Toledo.}

\footnotetext{
${ }^{\S}$ Estas dos obras fueron transcritas para la elaboración del trabajo fin de master del cual nace este articulo.

** Aunque hubo 4 opositores (Diego de Bruceña, , Francisco Bustamante, Lucas Tercero, y Joan Siscar) estos no convencieron al cabildo ni a sus músicos.
} 


\section{JUAN BAUTISTA CANADILLAS GÓMEZ}

Prácticamente nada se conserva de la actividad compositiva de este autor, únicamente una obra que aparece en el Códice Polifónico $n^{\circ} 25$, un himno titulado Jesu Corona Virginum, a 4 voces, que fue transcrito y editado por el hispanista Michael Noone (2003: 137). Además como se cita previamente existe un Magnificat a 8 voces conservado a papeles sueltos, cuya autoría podría pertenecer a este maestro.

\section{Los Organistas de la Catedral de Toledo}

Se puede presuponer que el organista es aquel instrumentista dedicado a la interpretación musical con el órgano. Sin embargo, la catedral de Toledo, con sus Actas Capitulares y sus libros de Obra y Fábrica genera un problema en la terminología. Si bien las Actas Capitulares hacen uso del término organista de forma más o menos profusa e indiferenciada para los distintos músicos intérpretes de órgano (ACCT, Vol. 23, AC de 2412-1604, Fol. 250v y ACCT, Vol. 24, AC de 01-09-1607, Fol. 147r.), en los libros de Obra y Fábrica este término aparece muy bien diferenciado del de músico de tecla. Esta última acepción aparece empleada para el racionero Jerónimo Peraza, para Gregorio Peñalosa y en una fecha más tardía (de 1607 en adelante) para Juan Peraza; entre tanto el término organista es empleado únicamente hasta 1608 para Melchor de Miranda. Ello podría significar que bien existía una diferenciación jerárquica entre los organistas en base a la dicha denominació o que en la Obra y Fábrica se emplee organista como sinónimo de organero; una información extraída del partido de libramientos de los libros de Obra y Fábrica del año 1608 donde se premia a Melchor de Miranda por el "Aderezo de los órganos grandes y pequeños" en tres ocasiones parece apuntar en esa dirección.

En el rango de fechas que abarca este trabajo encontramos 3 organistas que trabajaran para la catedral, además de los múltiples que trabajarían en las diferentes capillas como la de Don Pedro Tenorio o en la de San Pedro. Estos 3 músicos de tecla serían:

\subsection{JERÓNIMO PERAZA}

Su fecha de nacimiento es desconocida, nacido en Toledo, hijo del ministril de chirimía y corneta de la catedral Juan Peraza, "el viejo", quien se mantuvo en este puesto hasta el año 1570 (Reynaud, 1996: 259). Su madre, Juana Moreno era también según las informaciones una reputada instrumentista (López-Calo, 1999: 603).La familia, como era común por otra parte en la época, se dedicó casi por completo a la música, su hermano Francisco fue organista como él y su hermano Juan ocupó el mismo puesto que su padre.

En 1578, con la muerte del organista Juan de Peñalosa, Jerónimo entendió que era el momento idóneo para tratar de ocupar el puesto en la primada de Toledo y dejar el que poseía en Sevilla.

Las audiciones toledanas se llevaron a cabo delante de los canónigos capitulares quienes invitaron a asistir al anciano maestro de capilla y a tres cantores, quienes se encargarían de decidir las piezas que debían tocar para mostrar su habilidad. Peraza saldrá airoso de esta prueba por un justo margen, pero además inmediatamente después presentó un escrito en el cual se comprometía a servir por el resto de sus días a la catedral (Reynaud, 1996: 181). 


\section{La CAPILla musical de la CATEDRaL DE TOLEDO DURANTE EL PRIMER DECENIO DEL SIGLO XVII}

Tomará posesión de su ración en marzo de 1580, y recibirá un salario de 200 ducados (75.000 maravedís). En el año 1602 enterado el cabildo de los ofrecimientos que le llegaban a su racionero por parte de otras catedrales (entre ellas la de Sevilla), decide darle prevalencia a la hora de ocupar las capellanías (ACCT, Vol. 23, AC de 26-03-1602, Fol. 18v.).

A partir del año 1603 las noticias más comunes que se encuentran a cerca de este organista son aquellas referidos a sus permisos. También se hace alusión a su mandato de informar a cerca de un opositor a organista de capilla (ACCT, Vol. 24, AC de 08-06-1606, Fol. 42v), se le menciona en a la hora de admitir a su sobrino, o en una solicitud para la compra de un majuelo. La última noticia que se tiene sobre él en el periodo que abarca esta investigación, es un permiso para ir a su vendimia en la villa de Ajofrín (ACCT, Vol.25, AC de 12-10-1609, Fol. 91v).

\subsection{Gregorio de PeÑalosa}

En mayo de 1585 Gregorio de Peñalosa es nombrado ayudante de organista con un salario de 30.000 maravedís (Reynaud, 1996, p. 186), sobre el cual, en el año 1592, recibirá una gracia del cabildo que lo aumentará hasta 50.000 maravedís, este se mantendrá estable hasta que el 10 de Abril de 1604 reciba un nuevo aumento que lo dejará con un salario anual de 60.000 maravedís, el cual mantendrá hasta el final del periodo a estudio ${ }^{\dagger t}$.

Además de esta información existen pocas noticias que hagan alusión al músico, si bien las existentes hacen referencia a que el cabildo tenía en consideración la "opinión profesional" del mismo a la hora de determinar cuestiones relacionados con la concesión de puestos de organista (ACCT, Vol. 24, AC de 31-05-1606, Fol. 38r).

\subsection{JUAN PERAZA}

La primera alusión a este instrumentista la encontramos en los libros de Obra y Fábrica de 1607, se menciona que su contratación tuvo lugar el 10 de Junio del dicho año, y se le recibió con un salario igual al del racionero-músico de tecla Jerónimo Peraza. Sin embargo las Actas Capitulares no reflejan para el dicho día la contratación de ningún músico, sí que lo hacen el 28 de Mayo, de un instrumentista de sacabuche llamado Juan Peraza:

El dicho día llamados para tratar si se recibía en esta Santa Iglesia por músico de sacabuche a Joan Peraza sobrino del Racionero Hierónimo de Peraza y con qué salario y habiendo votado sobre ello mandaron al señor Don Gerónimo de Miranda suplique a su Ilustrísima. Le mandaron señalar 200 ducados de salario en la obra (ACCT, Vol. 24, AC de 26-05-1607, Fol. 125v).

\footnotetext{
${ }^{++}$Además de este salario recibía una ayuda de costa de 10.000 maravedís.
} 


\section{JUAN BAUTISTA CANADILLAS GÓMEZ}

Como se puede ver las fechas aproximadamente coinciden y el individuo responde al mismo nombre pero sin embargo, no encontramos concordancia en la labor que llevan a cabo. En tiempo posterior no se hace ninguna referencia a la cuestión, ni a si fue un error. El resto de alusiones a él en el periodo de estudio son los libros de Obra y Fábrica de los años 1607, 1608 y 1609 donde se le menciona como músico de tecla.

\section{LOS SOCAPISCOLES}

Los socapiscoles tuvieron un papel determinante en la ejecución musical de la catedral. Existían en Toledo dos raciones para este cargo, además había también ayudantes de socapiscol encargados de asistirlos en sus labores y en caso de ausencia de sustituirlos; estos individuos solían ser cantores de música polifónica que para ganar un sobresueldo se realizaban este trabajo(ACCT, Vol. 23, AC de 26-08-1602, Fol. 43r).

Durante la década de 1600-1609 las raciones de socapiscoles fueron ocupadas por Antonio Sancho y Juan Rojo. Y la labor de ayudante de los mismos fue llevada a cabo por Alonso de Torres y posteriormente por Eugenio Campos. Existe una noticia que alude al nombramiento como ayudante de socapiscol de Joan Corral (ACCT, Vol. 24, AC 02-091606, Fol. 76r) sin embargo los libros de Obra y Fábrica no reflejan ninguno de los aumentos usuales para estos individuos.

Si bien encontramos un número considerable de permisos de distinto tipo como para ir a su tierra o algunos sin especificar, y también aparecen noticias referentes a aumentos de salario, son mucho más habituales otro tipo de referencias relacionadas con la actividad musical como la búsqueda de cantores (ACCT, Vol. 24, AC de 24-09-1608, Fol. 230r), el examen de aptitud de los mismos y de los salmeadores (ACCT, Vol. 24, AC de 0502-1607, Fol. 107r), el de los socapiscoles de maitines y el de los maitinantes (ACCT, Vol. 24, AC de 20-10-1608, Fol. 236r); el mantenimiento de cantores que venían a examinarse (el hospedaje no era únicamente realizado por ellos); y por último la corrección y revisión de libros de canto (ACCT, Vol. 22, AC de 27-03-1604, Fol. 161).

\section{El Maestro de Música del Colegio de los Infantes}

En esta década, el titulo de maestro de música del Colegio de los Infantes y el de claustrero generan cierta confusión. El puesto de claustrero poseía ración desde el año 1448, cuando una bula de Nicolás V autoriza la creación de la misma (Preciado, 1974: 82). Por su parte el Colegio de los Infantes fue creado en el año 1562 (Reynaud, 1996: 29) y junto a él su maestro de música. Estos dos cargos se encontrarán por separado hasta que en el año 1599 Juan de la Peña los posea; primeramente en abril de 1597 adquirió el de maestro de música del Colegio de los Infantes, abandonando su puesto como maestro de capilla de la catedral de Cuenca (Preciado, 1974: 82) y después en 1599 tomó el de claustrero sustituyendo a Juan Rodríguez.

Durante el periodo 1600-1609 se encuentra activo en ambos cargos y por ello las Actas Capitulares producen dudas debido a los títulos con los que se refieren a él. Su cargo no quedaba únicamente restringido a la enseñanza de los jóvenes, tanto en el colegio como en la catedral, puesto que se le encargaban multitud de labores, algunas usuales entre los 


\section{La CAPILla musical de la CATEDRaL DE TOLEDO DURANTE EL PRIMER DECENIO DEL SIGLO XVII}

músicos racioneros como la búsqueda de cantores o la búsqueda de seises (ACCT, Vol. 24, AC de 11-12-1606, Fol. 91r); otras más específicas respecto a su puesto de trabajo como sería llevar el compás de la melodía "a los muchachos"( ACCT, Vol. 23, AC de 23-03-1604, Fol. 160v) y por último las que denotaban la confianza que el cabildo depositaba en sus habilidades como sería la corrección de los libros de canto llano, labor en la cual el cabildo no consintió la intromisión de ninguna otra persona (ACCT, Vol. 23, AC de 23-03-1604, Fol. 160v), o la composición de la sibila, por la cual se le ofrecía una remuneración económica.

\section{LOS CANTORES}

En el caso de Toledo existían dos de estos racioneros por cada "cuerda" (Reynaud,

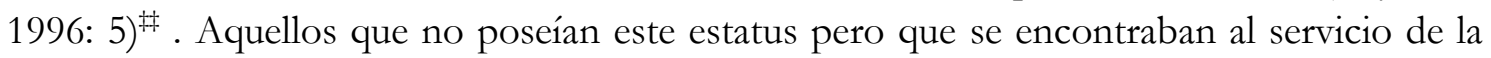
iglesia se denominaban asalariados; la cuantía económica que recibían por su labor variaba en función de distintos factores como la habilidad. Para los salmeadores no existían raciones por lo que todos ellos fueron asalariados.

El reclutamiento de los cantores racioneros se llevaba a cabo de la misma forma que la del resto; una vez que la ración vacaba bien por abandono bien por muerte, el cabildo ordenaba que se pusiesen los edictos para el puesto; los cuales daban 60 días para presentarse a una oposición por él. Tras este paso vendría la examinación; el hospedaje de los opositores solía ser encargado algún miembro de la capilla de música. A continuación se decidía si el cantor era óptimo para ocupar la plaza y se realizaban las averiguaciones de limpieza de sangre. Una vez finalizada se exponía ante el cabildo quien la verificaba y concedía la provisión de la ración.

La otra manera de proveer un puesto de cantor era la búsqueda directa, en la cual, el cabildo encargaba a algún racionero que viajase a buscar las voces necesarias. En ocasiones se aprovechaba la marcha de alguno de los músicos de la catedral a su tierra para buscar miembros para la capilla (ACCT, Vol. 24, AC de 09-06-1606, Fol. 43v-r). También encontramos solicitudes muchísimo más precisas donde se pide que el comisionado para dicha tarea visite determinados lugares, normalmente debido a su importancia(ACCT, Vol. 22, AC de 10-04-1600, Fol. 225r); existen incluso solicitudes en las cuales se pide que se vaya a ver la habilidad de un cantor muy concreto, como es el caso de Joan Sanz, tenor de la catedral de Burgos(ACCT, Vol. 22, AC de 27-08-1601, Fol. 328v). Una vez realizados estos trámites y si los mismos estaban de acuerdo con las condiciones ofrecidas por la catedral de Toledo, se procedía a recibirlos con un salario en la Obra.

El cabildo fomentó la superación y el avance a través del estudio de sus músicos, permitiendo licencias para ello (ACCT, Vol. 22, AC de 08-08-1600, Fol. 252v) y concediendo aumentos de salario.

\footnotetext{
‡¥ Este aumento se llevó a cabo por la solicitud del Cardenal Jiménez de Cisneros al Papa Alejandro VI en 1549.
} 


\section{JuAN BAUTista CANADillas GÓMEZ}

Las plazas existentes para cantores en la catedral eran limitadas, sin embargo, se dieron casos en los cuales se concedió a algún músico una plaza extraordinaria:

El dicho día estando los dichos señores capitularmente ayuntados todos unánimes y conformes recibieron a Alonso Díaz, natural de Vargas por Psalmeador en plaza extraordinaria con cuarenta y seis mil maravedís de salario por horas y cometieron al señor Don Dionisio de Melgar lo suplique a su señoría Ilustrísima; lo tenga por bien y mande asentar el salario en los libros de obra (ACCT, Vol. 22, AC de 31-101600, Fol. 270v).

\section{LOS MINISTRILES}

Son raras las referencias a cerca de los instrumentos utilizados por cada uno de los músicos, utilizando para aludir a ellos tan solo el título de ministril; es cierto que se menciona en el momento de la contratación, pero debido a las pocas ocurridas durante la década 1600-1609 es difícil de rastrear sin apoyarse en otros materiales. Sin embargo Reynaud en su tesis doctoral sobre la capilla musical durante el siglo XVI recopila prácticamente todas las especialidades de cada uno de los ministriles que conectan con este periodo. Es interesante también observar, que todos los que sirvieron a la catedral y fallecieron durante este tiempo, lo hicieron al servicio de la misma, lo cual podría indicar que las condiciones contractuales para ser admitido en este puesto continuaban vigentes ${ }^{\mathbb{S}}$.

\subsection{LOS INSTRUMENTOS}

Aparece una única mención a la compra de instrumentos para el periodo 16001609, pero esta ya fue recogida por Reynaud en su obra La polyphonie tolédane et son milieu, des premiers témoignages aux environs de 1600 por lo cual la información expuesta en este apartado está recogida de la misma. Los instrumentos empleados podían ser de dos tipos; unos pertenecientes a la catedral y otros de carácter personal.

Los instrumentos personales. Son bien aquellos que los músicos habían venido empleando con anterioridad o bien los que por cuestiones de necesidad adquirieron durante su trabajo al servicio de la catedral. Estos instrumentos eran comprados usualmente gracias a los préstamos que el cabildo le concedía para tal fin, ejemplo es el préstamo hecho a Gaspar Villegas en el año 1600 de 6800 maravedís para la compra de otra chirimía y del cual fue garante el también ministril Francisco de Aragón (Reynaud, 1996: 210).

Los instrumentos pertenecientes a la catedral. Estos instrumentos serían empleados por los ministriles como una suerte de préstamo mientras se encontrasen al servicio del cabildo teniendo que ser devueltos en caso de marcha o muerte. De los mismos no hay alusiones directas en la primera década del s. XVII, pero existen múltiples menciones en el

\footnotetext{
${ }^{\S}$ Como por ejemplo tener que servir al menos 25 años en la catedral.
} 


\section{La CAPILla MUSiCAL DE la CATEDRAL DE TOLEdo DURANTE EL PRIMER DECENIO DEL SIGLO XVII}

periodo previo, destacando especialmente la compra de flautas; cuya primera adquisición, en un número de 8, el cabildo llevó a cabo en el año 1531 (Reynaud, 1996: 208).

\section{OTROS INSTRUMENTISTAS}

Además de los ministriles que se encontraban al servicio pleno de la catedral, existían otros que excepcionalmente llevaban a cabo actividades para la iglesia, siendo la más habitual la interpretación para las festividades del Corpus, su Octava, la de Nuestra Señora de la Asunción y su Octava. De estos músicos apenas quedan noticias más allá de las respectivas libranzas económicas por sus servicios; aun así podemos diferenciar diversos tipos de instrumentistas que participaron en las dichas actividades como los tañedores de violones (como Rodrigo de Ayllón), "clarinetistas" como Andrés del Buen Homo y sus compañeros (ACCT, Vol. 22, AC de 05-06-1600, Fol. 239v), trompetistas (de lejos los más usuales) como Juan Carrera o Diego Cantero (ACCT, OF año 1609, Partido de danzas y autos, 4 de Julio) o interpretes de chirimía (ACCT, Vol. 24, AC de 07-06-1606, Fol. 41r). Un caso interesante es el de la noticia que hace referencia a la procedencia de los instrumentistas, en este caso llamados "catalanes" y que ofrecieron sus servicios aparentemente como un grupo conformado (ACCT, Vol. 24, AC de 18-08-1607, Fol. $139 \mathrm{v})$.

\section{LOS SEISES}

La denominación de seise viene referida no sólo al número en el que se fundaron, sino también en el que se consideraba más idóneo para su correcto cuidado y aprendizaje, además de para su uso en las iglesias. Sin embargo este valor no fue absoluto, y en base múltiples cuestiones como la mudanza de la voz, las faltas de disciplina (Martínez Gil, 2003: 142) o la aparición de un joven con una calidad interpretativa fuera de lo normal (Reynaud 1996: 142-143) podemos encontrar un número mayor o menor de ellos.

La primera vez que aparece citado este término en la documentación de la catedral de Toledo es el año 1461, cuando se le encarga a un maestro que los mantenga, los instruya musicalmente y los eduque. Anteriormente habían estado considerados en conjunto con el resto de los clerizones, aunque sus funciones hubiesen sido ligeramente diferentes.

La fórmula para convertirse en seise podía llevarse a cabo de dos maneras; la primera y más usual por presentación directa a la catedral con intención de servir como tal y la otra mediante su captación en los viajes en busca de cantores.

Una vez superadas todas las pruebas, eran encargados al maestro de capilla para que llevase a cabo su instrucción y dispusiese de ellos. Lo usual es que habitasen en el hogar del maestro o maestro interino. Además de aportarles alojamiento, se encargaba de alimentarlos, por estos servicios recibía una cantidad preestablecida pero variable dependiendo del número de niños y cobrada por tercios. Además el cabildo realizaba anualmente provisiones para sus ropas aunque de forma excepcional podían realizar libranzas atendiendo a la necesidad(ACCT, Vol. 25, AC de 24-04-1609, Fol. 34v). Dentro de los cuidados ofrecidos a los jóvenes se encontraban aquellos relacionados con la salud 


\section{JUAN BAUTISTA CANADILLAS GÓMEZ}

realizándose pagos anuales a médicos (ACCT, Vol. 24, AC de 07-02-1607, Fol. 108v) y boticarios(ACCT, Vol. 22, AC de12-01-1600, Fol. 203v).

En el periodo de estudio comprendido por este trabajo, 19 jóvenes de diferentes procedencias se presentaron para ocupar estos puestos, de los cuales fueron admitidos 11 en diferentes años: uno en 1600, cuatro en 1601, dos en 1603, uno en 1604, otro en 1605, otro en fecha desconocida (aparece en un libramiento de 1608) y otro más en 1609. Como se puede ver el flujo de entrada ( $\mathrm{y}$ de salida por contrapartida) de chicos fue constante durante estos años.

Los jóvenes cantores recibían enseñanzas de gramática y latín impartidas por el maestroscuela y lecciones de canto instruidas por el claustrero al igual que los clerizones sin embargo debían de esforzarse también en el aprendizaje del canto polifónico, el cual dirigía el maestro de capilla .

También desarrollaban otro tipo de actividades por las cuales les eran ofrecidas diferentes tipos de gracias; un ejemplo de esto sería la intervención en las danzas(ACCT, Vol. 23, AC de 30-08-1605, f. 309v). Sin embargo, cabildo no concedió estas "gracias" únicamente en base a actividades realizadas sino también por razones como el despido por cambio de voz (ACCT, Vol. 23, AC de 22-04-1605, f. 277r), al igual que a los adultos para viajar a su tierra, o incluso atendiendo a pobreza y necesidad.

\section{SALARIOS}

Los salarios recibidos por los músicos al servicio de la catedral fueron muy variados y dependieron esencialmente de 3 cuestiones: el estatus en la jerarquía musical, la habilidad interpretativa y el "grupo" del cual formaban parte (cantores, salmeadores, ministriles). Respecto a los sueldos es necesario mencionar que se cobraban por tercios, a excepción de los ministriles quienes recibían sus cobros cada dos meses (algo estipulado en las condiciones de contratación (Reynaud, 1996: 204)). También es necesario aludir a que las cantidades a las que se hace referencia son absolutas, es decir, que de ellos habría que descontar préstamos, penalizaciones y faltas de asistencia para conocer el salario real que cada uno de los músicos percibió en este periodo.

El sueldo de los distintos maestros de capilla se mantuvo estable durante este periodo; ambos (Lobo y Tejeda) alcanzaron tras sendos aumentos un montante total de 200 ducados (75.000 maravedís), sin embargo no era la única remuneración que recibían ya que el cabildo solía gratificarlos por sus composiciones y como se mencionó previamente recibían a parte una cuantía por el cuidado y enseñanza de los seises.

En el caso de la organistía dependía en gran medida de la posición y de la habilidad. El racionero cobraba un salario de 200 ducados que se mantuvo inamovible durante esta década; por su parte los músicos de tecla recibieron cantidades bastante dispares, Gregorio de Peñalosa percibía en el año 160050000 maravedís que en 1604 aumentaron en 10000 más, en cambio Juan Peraza desde su contratación recibió un montante de 75.000 maravedís. Por su parte el "organista" Melchor de Miranda recibió por sus servicios durante todo el periodo una cantidad de 17.000 maravedís. 


\section{La CAPILla musical de la CATEDRaL DE TOLEDO DURANTE EL PRIMER DECENIO DEL SIGLO XVII}

Los 2 socapiscoles de la catedral no recibieron un salario uniforme, Antonio Sancho percibió 48.750 mrvs. hasta el año 1606 y finalizó la década cobrando 75.000 mrvs; su contraparte Juan Rojo comenzó recibiendo un salario de 12000 mrvs. y lo finalizó con uno de 40000. Los ayudantes de socapiscol recibían una cuantía que se añadía al salario que tenían como cantores, aún así los libros de Obra y Fábrica muestran los sueldos desglosados. Alonso de Torres percibió hasta el año 1602 la cantidad nada despreciable de 70.000 mrvs. por su función, Eugenio de Campos quién comenzó a servir en este puesto en 1602 recibía una cuantía de 25.000 mrvs. que se mantuvo estable hasta donde alcanza este estudio.

El salario del maestro de música del Colegio de los Infantes, Juan de la Peña, fue modesto en términos comparativos con los que se están barajando; recibía 17000 mrvs., además de 1000 mrvs. por componer la Sibila y otros 8000 por su trabajo como corrector de los libros de coro (a partir de 1605).

El sueldo percibido por los cantores fue muy variado y en gran medida dependiente de las habilidades musicales del individuo. Por regla general cobraban más que los salmeadores sin embargo no ocurría de la misma manera entre los racioneros (cantores) y los asalariados; como ejemplo Jorge de Santa María poseía ración y percibía 20000 mrvs por su labor mientras que un Joan Corral como asalariado cobraría 102.000 mrvs desde el inicio de las indagaciones (además a partir de 1603 cobraría también por guarda de los libros del coro $7500 \mathrm{mrvs})$.

Finalmente el caso de los ministriles reviste una interesante peculiaridad y es debido a que fueron los poseedores del mayor salario entre los músicos de la catedral. Es cierto que no todos ellos percibían cantidades desorbitadas pero en términos generales la media superó, con mucho, a la del resto. Sirva de ejemplo el caso de Gaspar de Villegas quien recibía un salario de 45000 mrvs para el año 1600 alcanzando al final del periodo de estudio 84500 mrvs. Además el salario más alto percibido por un músico en la catedral fue de un ministril, el de Juan Peraza, hermano del racionero, quien mantuvo un salario de 195.000 maravedís desde el año 1600 hasta el 1609. Los ministriles además poseían dos ayudas de costa anuales e incluso algunos de ellos una tercera.

\section{PeRmisos y CoNCESIONES}

La catedral tenia en una gran estima a sus músicos y por ello en muchas ocasiones "no haciendo falta en el coro" les concedía permisos de carácter variado. Son las menciones a estos permisos de diferente carácter las noticias más usuales que encontramos respecto a los miembros de la capilla en las Actas Capitulares de la catedral.

La siguiente agrupación de licencias ha sido creada únicamente con la intención de poder aglomerar el gran y variado número de las mismas existentes.

\subsection{LICENCIAS PARA IR A OTRA IGLESIA O CATEDRAL}




\section{JuAN BAUTisTa CANADiLlas GÓMEZ}

Estos permisos daban el consentimiento del cabildo para que los músicos (cantores/ministriles) llevasen su labor musical al servicio de otro centro religioso. Pero las condiciones eran muy explicitas, se decía el lugar al que se les permitía marchar, el número de ellos que podían hacerlo y el momento o periodo en el cual podían llevarlo a cabo; todo ello siempre supeditado a su necesidad en el coro catedralicio. Usualmente los centro a los cuales iban eran de un orden inferior y se encontraban situados o en la misma capital o en las proximidades si bien había excepciones como el caso de la solicitud llegada desde la catedral de Ávila:

El dicho día e recibió una cuenta del Cabildo de Ávila en que da cuenta a este lugar como su Majestad va a aquella ciudad para doce de Junio y tienen necesidad de algunos cantores de esta Santa Iglesia y de algunas cosas del sagrario y habiendo visto la dicha carta tratado y conferido sobre ello, mandaron que los Racioneros Miranda y Guzmán y Gerónimo López y Peraza, ministriles, vayan a la dicha ciudad en esta ocasión y que el señor Deán oiga a Joan Yáñez Loares racionero de aquella iglesia que trajo la carta y le mande dar del sagrario algunas cosas que hayan menester y responda a la carta (ACCT, Vol. 22, AC de 31-05-1600, Fol. 237r).

\subsection{LICENCIA PARA IR A SERVIR A LA ARISTOCRACIA}

Existe una única mención durante este periodo a un permiso de este tipo sin embargo dicha licencia ofrece información bastante relevante puesto que su concesión nos habla de un vínculo entre la catedral y el noble en cuestión: "El dicho día estando los dichos señores capitularmente ayuntados dijeron que los cantores que pide el señor Marqués de Villena y todo lo demás de tapicería y blandones se le dé y que el señor Deán lo mande cumplir" (ACCT, Vol. 22, AC de 17-10-1601, Fol. 338v).

\subsection{PERMISOS PARA MARCHAR A SU TIERRA}

Por ellos se permitía poder abandonar la ciudad y por tanto el servicio de la catedral por un tiempo especificado(el cual variaba en función de factores como la época del año o la lejanía de la localidad) para ir a su lugar de origen. Estos permisos son los más usuales.

\subsection{PERMisos POR CUESTIONES VARIADAS}

El cabildo era bastante considerado con sus músicos, si se encontraban enfermos realizaba concesiones para su no asistencia al coro (ACCT, Vol. 23, AC de 16-06-1603, Fol. $92 v$ ). Si tenían viñas y debían recogerlas también solían hacerles gracia de la asistencia, podían ir de romería y les permitía asistir a las honras de un pariente como muestra la siguiente noticia: "Este día dieron licencia a Joan Fernández, tiple, cantor de esta Santa Yglesia para que vaya a Párragas, de donde es natural a hacer honras por su madre con que vuelva para el día del Corpus de este año" (ACCT, Vol. 23, AC de 22-05-1609, Fol. 51v).

\subsection{PERMISOS SIN ESPECIFICAR}




\section{LA CAPILla MUSical de la CATEDRAL DE TOLEDO DURANTE EL PRIMER DECENIO DEL SIGLO XVII}

Este grupo de permisos engloba a todos aquellos en los cuales tenemos noticia de la concesión de la licencia para ausentarse del coro pero no existe más información al respecto (ACCT, Vol. 23, AC de 07-09-1602, Fol. 46v).

\section{Conflictos}

En todo el periodo a estudio la conflictividad arrojada por las Actas Capitulares para los músicos de la capilla es relativamente baja, tan solo encontramos 6 conflictos que afecten a alguno de ellos. Es importante referir que esta cifra puede ser "ficticia" ya que con total seguridad el cabildo no tendría la necesidad de intervenir en todos las disputas existentes y por tanto estos no habrían quedado reflejados De ello podría ser muestra la siguiente noticia : "El dicho día cometieron a los señores Doctores Espinosa y Tena y Don Francisco de Monsalve traten que entre los socapiscoles y cantores haya mucha paz y hermandad" (ACCT, Vol. 23, AC de 28-11-1602, Fol. 57v).

Es necesario decir también que 3 de esos 6 conflictos tuvieron como parte "agravante" a un mismo individuo el socapiscol Antonio Sancho, el primero ocurrió el 25 de Febrero del año 1600 y le enfrentó con el cantor Joan Corral (ACCT, Vol. 22, AC de 2103-1600, Fol. 222v); los dos siguientes ocurrieron en un periodo muy breve, uno el 5 de Abril de 1609 (ACCT, Vol. 25, AC de 06-04-1609, Fol. 31v) y el otro el 7 de Abril del mismo año (ACCT, Vol. 25, AC de 07-04-1609, Fol. 31v).

En 4 de los conflictos participó algún cantor pero nuevamente más interesante es que en 3 de ellos participó el mismo, Joan Corral. Además de la disputa mencionada antes, mantuvo otras dos, una con el cantor racionero Miguel de Mencos donde fue la parte "afrentada" por lo que no se le impuso pena alguna (ACCT, Vol. 23, AC de 07-05-1605, Fol. 281v) y con Eugenio de Campos donde la pena fue igual de severa para ambos ( ACCT, Vol. 24, AC de 02-09-1606, Fol. 76r.).

Por su parte el grupo de los ministriles solo presentó un conflicto sin embargo de una gravedad considerable; ocurrió en el año 1602 entre el intérprete de sacabuche Martín Pretel y el racionero cantor A. Segura. Tras las averiguaciones se decide despedir al instrumentista, sin embargo menos de un mes después es reintegrarlo en su puesto gracias a la intervención de Don Martín Aldrese (ACCT, Vol. 23, AC de 13-12-1602, Fol. 59v).

Digno de reflexionar es que de los 6 conflictos 5 son copados por únicamente dos personas. Por lo cual sería más correcto hablar de elementos conflictivos que de tendencias conflictivas dentro de la capilla.

\section{EXPULSIONES}

Las expulsiones de músicos como refleja Dionisio Preciado en su investigación sobre el maestro de capilla Alonso de Tejeda eran "cosa novedosa"( 1974, p. 71); sin embargo no eran tan inusuales, cada cierto tiempo encontramos que tras algún conflicto o falta un músico era despedido, aunque usualmente solo durante un periodo de "castigo" tras el cual y con la consecuente solicitud de perdón era readmitido. Bien es cierto que no en todos los casos fue así, por ejemplo para la década 1600-1609 encontramos dos 


\section{JUAN BAUTISTA CANADILLAS GÓMEZ}

expulsiones sin readmisión posterior, la de Juan de Bellimar y la de Pedro Sánchez del Cerro (ACCT, Vol. 22, AC de 06-10-1600, Fol.165r).

\section{FIN DE LA VIDA AL SERVICIO DE LA CATEDRAL}

El número de menciones a este tema es bastante reducido, algo lógico si tenemos en cuenta el breve periodo que abarca este estudio. El final de la vida al servicio de la catedral hace alusión no solo a la muerte sino a cuestiones como la jubilación de uno de los integrantes, como seria por ejemplo el caso de Jerónimo López (ACCT, Vol. 23, AC de 10-05-1605, Fol. 282r) o la posibilidad de los músicos de solicitar que se les señalase un lugar de entierro dentro de la catedral, que en ocasiones fue designado con posterioridad al fallecimiento, un ejemplo de ello es el del salmeador Juan Bautista Martínez: "El dicho día encomendaron al señor Doctor Tena señale sepultura a Joan Baptista Martínez Psalmeador y mandaron llamar para hacer alguna limosna para su entierro". (ACCT, Vol. 24, AC de 0106-1607, Fol. 127v).

Además ocurría que si el individuo era demasiado pobre como para costearse el entierro la catedral se hacia cargo de los gastos del mismo. Las ganancias obtenidas en sus últimos momentos de servicio eran entregadas a sus familiares y si la situación de estos era de pobreza el cabildo podía decidir ofrecerles una limosna (ACCT, Vol. 24, AC de 02-061607, Fol. 127v).

No todos los fallecimientos quedaban registrados en las Actas Capitulares pero si que se quedaban registrados en la Obra y Fábrica. Por ejemplo así ocurrió con el racionero cantor Esteban Primo que falleció el 22 de Septiembre de 1607 y la mención que arrojan al respecto las Actas Capitulares es del año siguiente, el 12 de Marzo de 1608 donde se trata lo que debe reflejar la lápida de su sepultura(ACCT, Vol. 24, AC de 12-03-1608, Fol. 178v).

\section{INTERVENCiÓN DEL CABILdo SOBRE Cuestiones MusicAleS}

Es interesante la aparición de información de acerca de cuestiones interpretativas, estas noticias no son para nada abundantes ya que por regla general el cabildo no intervendría sobre ello; sin embargo en algunos casos fue necesaria su actuación para optimizar la labor encargada a la capilla de música. $\mathrm{Al}$ respecto encontramos en una entrada de las Actas Capitulares una recopilación de advertencias de cómo se han de llevar a cabo las ceremonias, entre las cuales encontramos algunas dedicadas a los músicos:

22- Y en que todos los músicos tiples canten como los demás en las procesiones y en las horas y que el señor presidente los pene sino cantasen, para que no se excusen diciendo que se les echa a perder la voz con el canto llano pues antes se les adoba con el ejercicio de ello (ACCT, Vol. 25, AC de 04-12-1609, Fol. 111r).

35- ... El señor presidente tenga cuidado de que los capiscoles y psalmeadores digan las horas con pausa y que el coro no empuje el verso hasta que haya acabado el dicho y que todos respondan con igualdad sin que haya prisa ni atropellamiento en el decir de los divinos oficios y que se haga guardar con rigor (ACCT, Vol. 25, AC de 04-12-1609, Fol. 113v). 


\section{La CAPILla musical de la CATEDRaL DE TOLEDO DURANTE EL PRIMER DECENIO DEL SIGLO XVII}

No son las únicas advertencias realizadas a los cantores e instrumentistas de la catedral en esta lista sin embargo si que son las únicas con un matiz interpretativo.

\section{CONCLUSIONES}

La intención principal con este proyecto es mostrar el funcionamiento de esta capilla en los albores del "barroco musical" para poder ver los entresijos que movían la maquinaria de una de las grandes capillas musicales del país. La elección de las fechas de inicio y fin sin atender a la cronología del maestro de capilla de turno viene fundamentada en mi consideración personal de que estos maestros si bien fueron determinantes, no fueron indispensables en el funcionamiento de las capillas musicales.

En base a lo observado podemos decir que durante este tiempo hubo 2 maestros de capilla y dos maestros interinos (se dividieron sus funciones) y en ningún momento la capilla dejó de desempeñar su actividad a la perfección, es más, una capilla como la de la catedral de Toledo poseía un número de individuos cualificados para llevar a cabo las labores compositivas (quizá las de más difícil sustitución) más que suficiente (no hay más que recordar que el maestro de música del Colegio de los Infantes fue previamente maestro de capilla en Cuenca).

También he tratado de poner en valor aunque sea de forma sintética a uno de estos "maestros interinos" Jorge de Santa María, un racionero cantor con un salario moderado poseedor de una biblioteca musical propia y que ejerció de maestro de capilla educando a los seises y componiendo para el cabildo en el tiempo en el que la plaza se encontraba vacante (e incluso en algunos momentos que no) durante el último cuarto del siglo XVI y el inicio del siglo XVII.

Otra de las intenciones que perseguía era observar la evolución musical del siglo a través de las obras conservadas del mismo, aunque al circunscribirse a un periodo tan breve fue mucho más complicado. Se conservan "pocas" composiciones del siglo XVII y aún menos que puedan ser adscritas (al menos presuntamente) a la década 1600-1609; lamentablemente las tres empleadas en el desarrollo del trabajo del cual nace el presente artículo no han podido introducirse aquí pero podemos decir de manera telegráfica que es una extensión de la música polifónica del siglo XVI ya que posee cualidades más cercanas al renacimiento que al barroco como por ejemplo la escasa pretensión armónica frente al desarrollo de la melodía. 


\section{JUAN BAUTISTA CANADILLAS GÓMEZ}

\section{BIBLIOGRAFÍA}

\section{Fuentes Documentales}

Actas Capitulares:

- Volumen 22 (1597-1601)

- Volumen 23 (1602-1605)

- Volumen 24 (1606-1608)

- Volumen 25 (1609-1610)

Libros de Obra y Fábrica; frutos y gastos desde el año 1600 al año 1609.

\section{Volúmenes}

Álvarez Pérez, J. M. (1959). "La polifonía sagrada y sus maestros en la catedral de León (siglos XV y XVI)". Anuario Musical, 14, 39-62.

Capdepón Verdú, P. (2013). "El Magisterio de Capilla en la época de Sebastián Durón" en P. Capdepón. y J.J. Pastor (eds.). Sebastián Durón (1660-1716) y la música de su época. Vigo: Academia del Hispanismo.

Cárdenas Serván, I. (1987). El polifonista Alonso Lobo y su entorno. Santiago de Compostela: Universidad de Santiago de Compostela.

López-Calo, J. L. (1999). "Peraza", en E. Casares (Dir.). Diccionario de la Música Española e Hispanoamericana, Vol. 8, p. 603. Madrid: SGAE.

Martínez Gil, C. (2003). La capilla de música de la catedral de Toledo (1700-1764): Evolución de un concepto sonoro. Toledo: Servicio de Publicaciones de la Junta de Comunidades de Castilla la Mancha.

Nelson, B. (2010). "A little known part-book from Toledo. Music by Morales, Guerrero, Jorge de Santa María, Alonso lobo and others in Barcelona, Instituto Español de Musicología, fondo reserva, MS 1". Anuario Musical, 65, 25-56.

Noone, M. (2003). Códice 25 de la catedral de Toledo. (L. Gago, Trad.). Madrid: Alpuerto.

Preciado, D. (1974). Alonso de Tejeda (ca.1556-1628) polifonista español. Obras Completas. Madrid: Editorial Alpuerto.

Reynaud, F. (1996). La polyphonie tolédane et son milieu, des premiers témoignages aux environs de 1600. París: CNRS Éditions.

Rubio Piqueras, F. (1922). "Música y músicos toledanos (Continuación)". Toletum, 13, 191 235.

Rubio Piqueras, F. (1924). "Códices Polifónicos de la S.I.C.P de Toledo". Toletum, 21-22.

Stevenson, R. (1961). Spanish Cathedral Music in the Golden Age. Los Ángeles: University of California Press. 
La CAPILla MUSiCAL DE la CATEDRAL DE TOLEdo DURANTE EL

PRIMER DECENIO DEL SIGLO XVII

Viñas Mey, C., \& Paz, R. (1951). Relaciones histórico-geográfico-estadísticas de los pueblos de España hechas por iniciativa de Felipe II: Reino de Toledo. Madrid.

Fecha de recepción: 04/11/2016

Fecha de aceptación: 11/12/2016 